\title{
Identifying potential therapeutic targets of a natural product Jujuboside B for insomnia through network pharmacology
}

\author{
Hongwei Dua ${ }^{a}$ Xinlei Zhao ${ }^{b}$ and Aihua Zhangc
}

\begin{abstract}
Knowledge of the interactome improves the understanding of disease metabolism. Biological information about interactions among genes and their protein products, computationally extracted in the context of SysBiomics, can hint at molecular causes of diseases, be essential for understanding biological systems, and provide clues for new therapeutic approaches. Quick and efficient access to this data have become critical issues for biologists. We have implemented a computational platform that integrates pathway, protein-protein interaction, differentially expressed genome and literature mining data to result in comprehensive networks for insomnia and intervention effects of Jujuboside $B(\mathrm{JuB})$. The interaction data were imported into Cytoscape software, a popular bioinformatics package for biological network visualization and data integration, for screening the central nodes of the network, exploiting functional study of the central node genes, exploring the mechanism of insomnia. Results showed that seven differentially expressed genes confirmed by Cytoscape as the central nodes of the network in insomnia had interactions, forming a complicated interaction network (77 nodes, 96 edges). Among gene nodes, HBA1, LEP, MAOA, PRNP, GHRL, CLOCK and SLC6A4 were verified as the genes with maximal differential expressions. Of note, we further observed that the HBA1, LEP, SLC6A4 and MAOA were JuB target genes. The interaction network of the differentially expressed genes, especially the central nodes of this
\end{abstract}

Received: 18 March 2014 / Accepted: 4 April 2014 / Published online: 5 May 2014

(C) Horizon e-Publishing Group

CITATION

Du H, Zhao X, Zhang, A. (2014). Identifying potential therapeutic targets of a natural product Jujuboside $\mathrm{B}$ for insomnia through network pharmacology. Plant Science Today, 1(2), 69-79. http://dx.doi.org/10.14719/pst.2014.1.2.26

AUTHOR AFFILIATION

a Heilongjiang Academy of Traditional Chinese medicine, Sanfu Road 142, Xiangfang District, Harbin, Heilongjiang Province, China.

b Heilongjiang Province Hospital, Zhongshan Road, Xiangfang District, Harbin 150036, Heilongjiang Province, China.

c Heilongjiang University of Chinese Medicine, Heping Road 24, Harbin 150040, China.

*CORRESPONDENCE: Prof. Aihua Zhang, Email: aihuaz@yeah.net Telephone \& Fax: +86-451-86053141 network, can provide clues to the insomnia, early diagnosis and molecular targeted therapy. Our findings demonstrate that the integration of interaction network in genomic space can not only speed the genome-wide identification of drug targets but also find new applications for the existing drugs.

Keywords: Interaction network; network pharmacology; Jujuboside B; insomnia; targets; differentially expressed genes

\section{Introduction}

SysBiomics approach seeks to comprehend the complexity of organisms by combining many different kinds of data (protein-protein and protein-DNA interactions, protein modifications, biochemistry, etc.) to create predictive models. In the era of SysBiomics, the focus on understanding complex organisms is shifting from studying individual genes and proteins towards the relationships between them (Gilchrist et al., 2006; Hopkins, 2008). These relationships are usually expressed in terms of various kinds of biological networks. Network among biomolecules is an intensive field of research in SysBiomics. Molecular interactions are the focus of many functional genomics studies, and they form a cornerstone of Systems biology research. Network models can reveal complex relationships in large data sets, and network topologies have been shown to share remarkably consistent features across diverse fields. Many studies have reported interesting biological findings from these networks, including the relationships between various statistical properties of a gene and its function at the molecular level based on networks (Tamble et al., 2011). Network pharmacology can make an impact at several points in the drug-development process: target identification, lead discovery and optimization, preclinical efficacy and safety assessment, and mode-of-action. Network diagrams are used for visualizing and understanding these interactions, interpreting high-throughput experimental data, generating hypotheses and sharing results (Zou, Luo, Wei, \& Yang, 2011). Increasing complexity of functional genomics data drives the development of methods and tools for data integration and visualization (Wu, Jiang, Zhang, \& Li, 2008). 


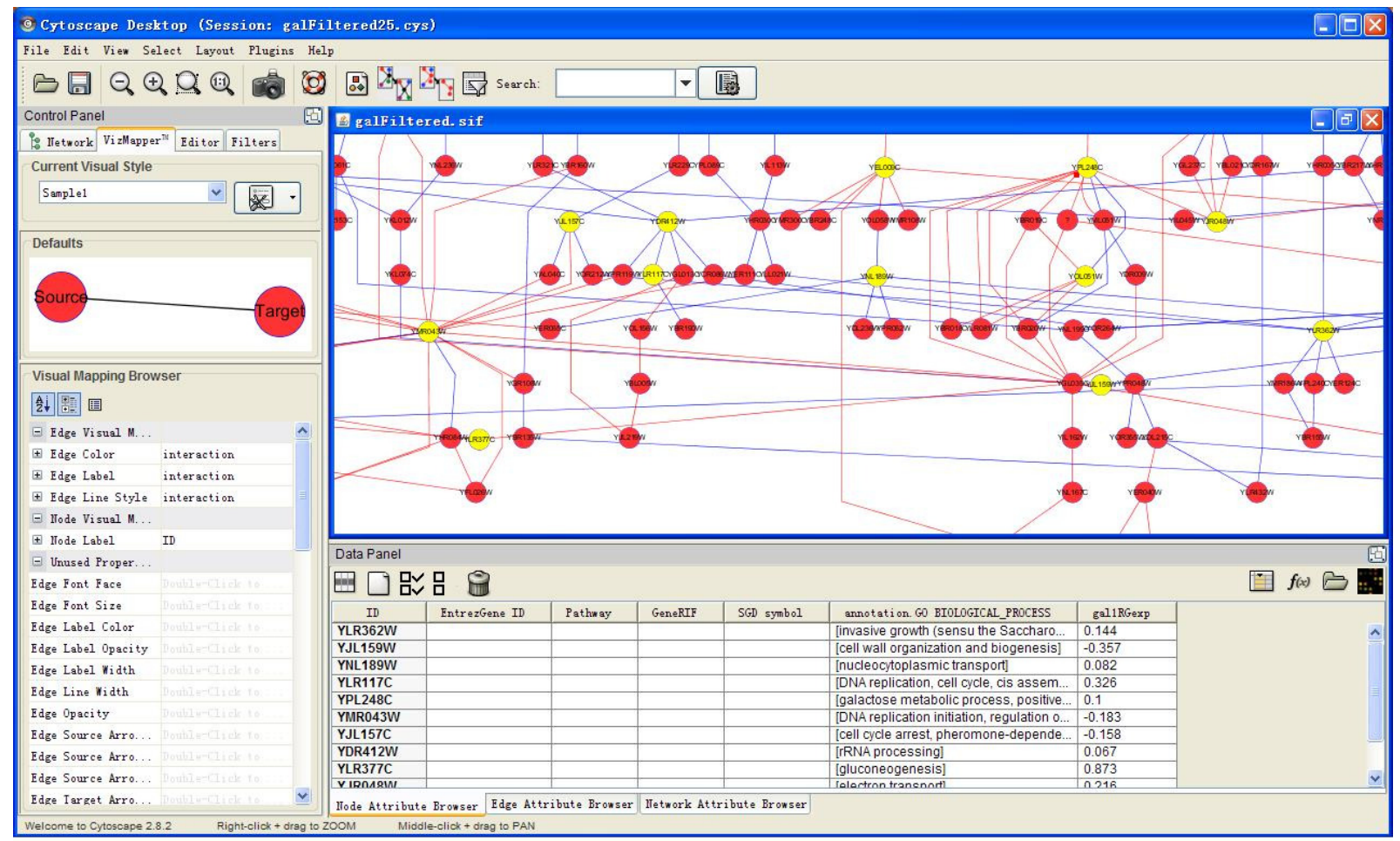

Fig. 1. Cytoscape version 2.8 shows a randomization instance of an integrated

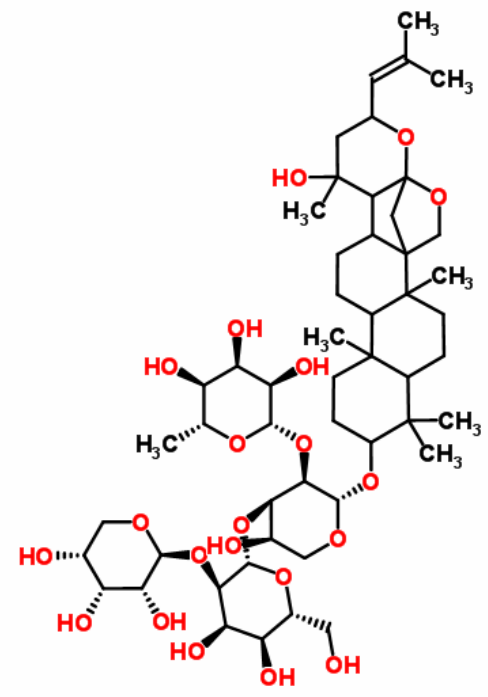

Fig. 2. Chemical structure of Jujuboside B

The most widely used open source network visualization workbench is Cytoscape which has greatly extended the reach of genomic research (Shannon et al., 2003). Data from various sources can be imported into this tool to build networks, and to highlight specific node or edge features. Cytoscape software (Fig. 1), a web-based network visualization tool, is an open-source software platform for visualizing molecular interaction networks and integrating these interactions with gene expression profiles and other functional genomics data. By providing platforms to integrate data with molecular interaction networks, researchers can more rapidly begin interpretation of large data sets collected for a system of interest. Currently, the network model is based on genes, proteins and functional relationships between them such as protein-protein, protein-DNA regulatory interactions and gene-protein coding relationship (Cline et al., 2007).

Disease gene prediction is one of the most important 


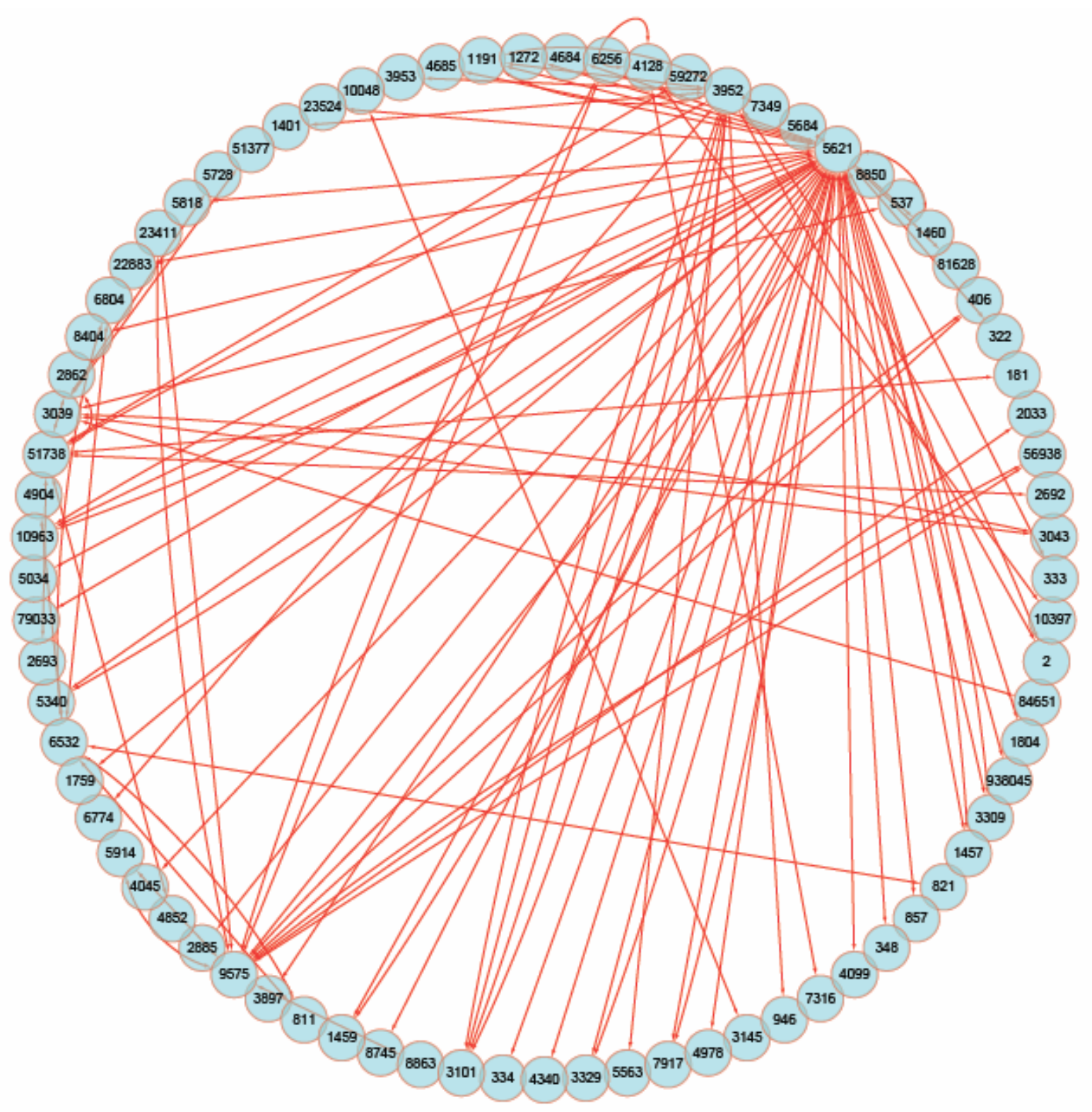

Fig. 3. Interaction network (Circle Layout) of the differentially expressed genes in insomnia using the Cytoscape

aims in biological and medical sciences. Network pharmacology has become more and more attractive in the research field of disease-causing gene discovery. Researchers attach great importance to special features embedded in biological networks especially the interaction network for deeply understanding molecular mechanism of common human diseases (Zhang, Shi, Duncan, Prodduturi, Marnett, \& Liebler, 2011; Yao, Hao, Li, \& Li, 2011). Network reconstruction of biological entities is very important for understanding biological systems. Together with network pharmacology, this preliminary study is one step toward genome-wide drug target identification. Until recently however, the analysis of insomnia network and its influence on disease-gene discovery are still unaddressed and insomnia model systems had little interaction data available.

Insomnia is a serious health problem, and enhancing sleep quality is an issue of significant importance to public health. Recently, it is estimated that more than one-third people in the world suffer from insomnia and frequently occurs at a rate of approximately 10 per cent in many countries and that causes a worldwide disease burden (Mahowald \& Schenck, 2005). Concerning sleep quality, $25.3 \%$ of the general population was dissatisfied with their sleep (Vecsey et al., 2009; Richey \& Krystal, 2011). Sedative-hypnotic drugs are increasingly prescribed for 

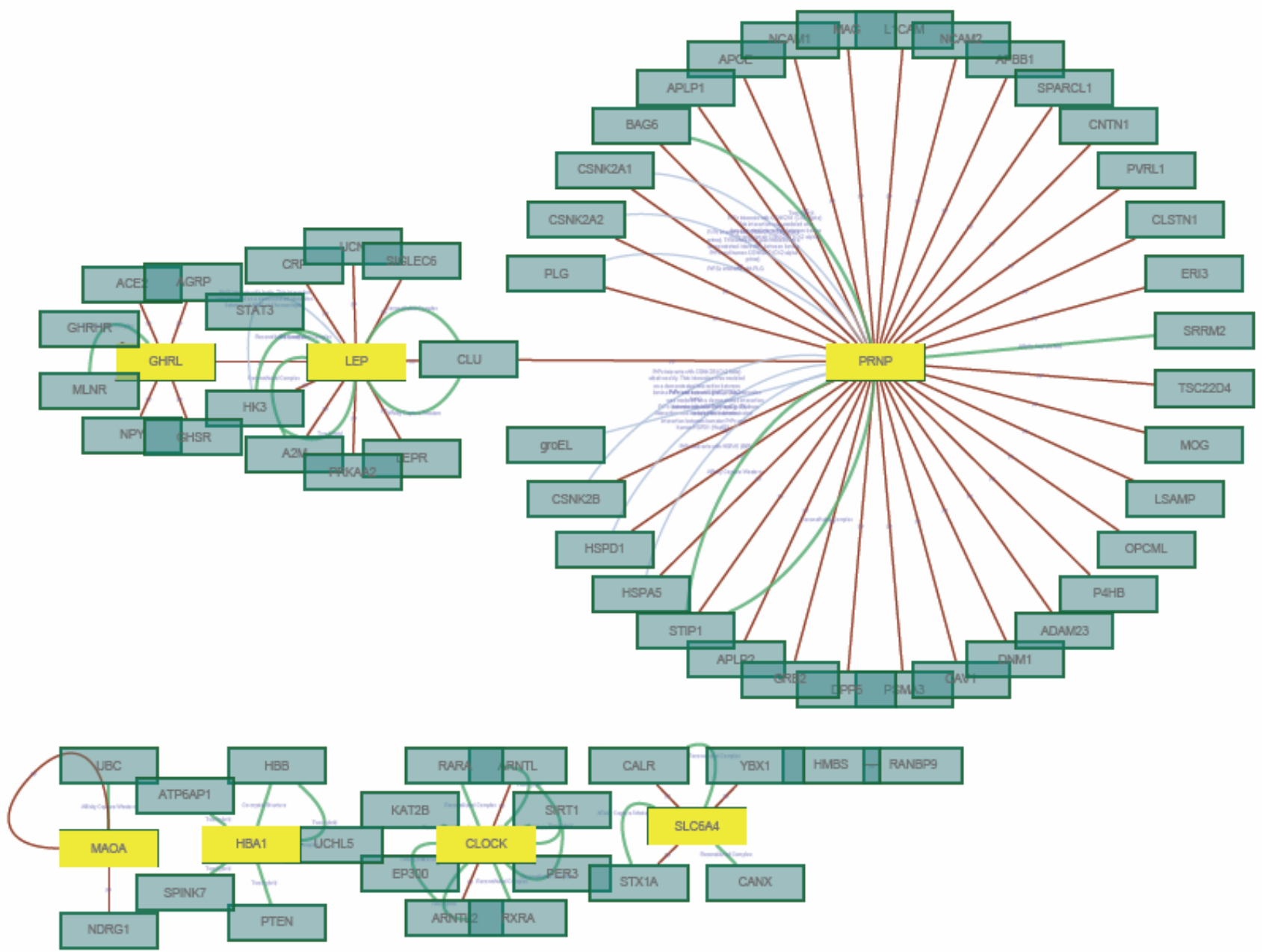

Fig. 4. Discrete insomnia network map with a customized visual Cytoscape Web style. Yellow nodes refer to the significant ontologies of the insomnia dataset

the insomniac patients, reflect the higher prevalence of anxiety and sleep disorders. However, a variety of problems, such as alteration of sleep structure, tolerance of the hypnosedative effects, pharmacological dependence, cognitive and psychomotor impairment, abuse potential and respiratory depression of these medications have drawn much attention. A growing body of evidence now suggests that these drugs do not exert satisfactory therapeutic effects (Sarris \& Byrne, 2011). The outlook is worsened by the absence of promising drug targets for this disease. Currently, a paradigm shift is occurring in that there is a new focus on the pursuit of new drug targets due to an urgent need for new drugs for the treatment of insomnia. More and more evidence supports that many Chinese medicinal products have been used for the treatment of insomnia and their therapeutic effects have also been verified by a host of clinical studies. Amusingly, a number of steroidlike compounds, such as triterpenoids, steroids and saponins, found in many Chinese medicinal products are used for promoting sleep regulation, and regarded as the active ingredients responsible for their therapeutic effects (Wang et al., 2008). Modern clinical studies have showed that suanzaoren, (Semen ziziphi spinosae), which is the dried seed of Ziziphus jujuba Mill var. spinosa (Bunge) $\mathrm{Hu}$ ex. H.F. Chou (Rhamnaceae), possesses multiple activities such as hypnotic-sedative, hypotensive, antihypoxia, antihyperlipidemia, and hypothermic effects (Fang, Hao, Zhou, Zhu, Wang, \& Song, 2010). A study indicates that suanzaoren exerts sedative effects mediated through GABA-A receptors (Zhao, Li, Yang, Li, \& Wang, 2006). Triterpene saponin Jujuboside B (JuB) (Fig. 2) extracted from suanzaoren is considered to be the major pharmacological active compounds responsible for insomnia treatment (Yang, 2012). The JuB has been shown to modulate sleep disruption in insomniac patients, however, its detailed targets mechanism for hypnotics function is poorly understood.

Reconstructing networks of biological system entities such as genes, transcription factors, proteins, compounds and other regulatory molecules is very important for 


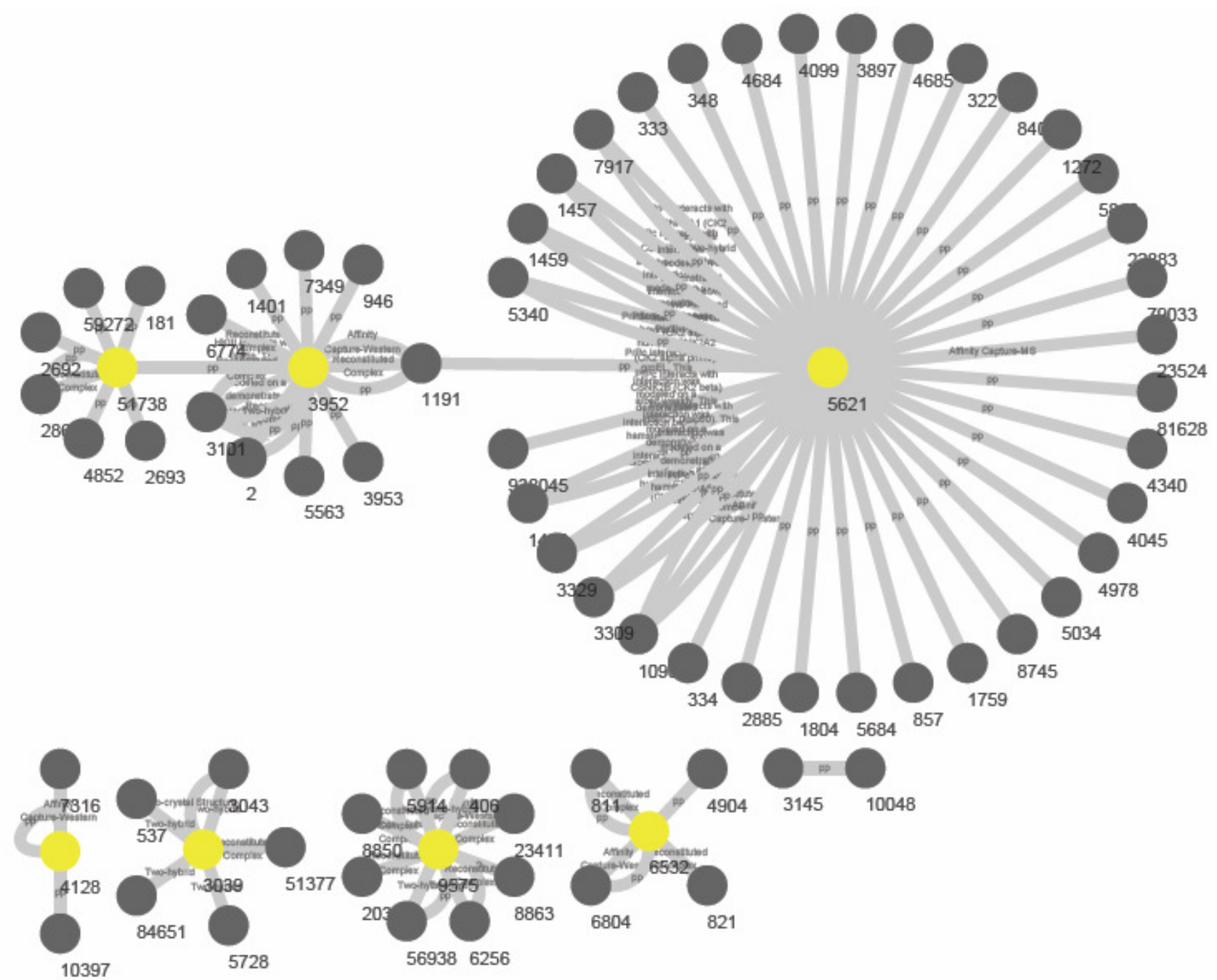

Fig. 5. Solid map on huge interactome of insomnia networks, built and visualized with Cytoscape. Edges: interactions. Nodes: specific proteins or genes

understanding the biological processes. Identifying drug targets is a critical step in pharmacology. Recent years, network pharmacology has influenced all areas of life sciences including that of drug mechanism and development, new target discovery (Janga, \& Tzakos, 2009). Efficient identification of drug targets is one of major challenges for drug discovery and drug development. Computational integration of different knowledge sources is a more effective approach and wealth of SysBiomics data provides unprecedented opportunities for drug target identification (Mestres, Gregori-Puigjané, Valverde, \& Solé, 2009). In types of networks, hubs are functionally important, and networks grow according to a preferential attachment model. Based on the correlations observed in pharmacological and genomic spaces, a computational framework, Cytoscape was developed to infer JuB-target interactions in a genome-wide scale. We highlight some capabilities of the Cytoscape with an exploration of insomnia pathogenesis genes, in which we identify a putative gene-drug discovery and exploration. In the present investigation, we give an illustrative example to show that the potential drug target identification problem can be solved effectively by our method, which may become an effective strategy for the discovery of new drugs for insomnia.

\section{Materials and methods}

Interaction information was retrieved from NCBI's Entrez Gene in December 2, 2011. Information on genes/proteins molecular function, biological processes and cellular component is imported from the Gene Ontology project; while information on biochemical pathways is taken from KEGG. Additional information includes links to databases, such as KEGG, Reactome, BioCyc, NCI Nature PID, DIP, BIND, HPRD, BioGRID, MINT, and Intact, which represent the major repositories of interacions from multiple organisms. The associations 

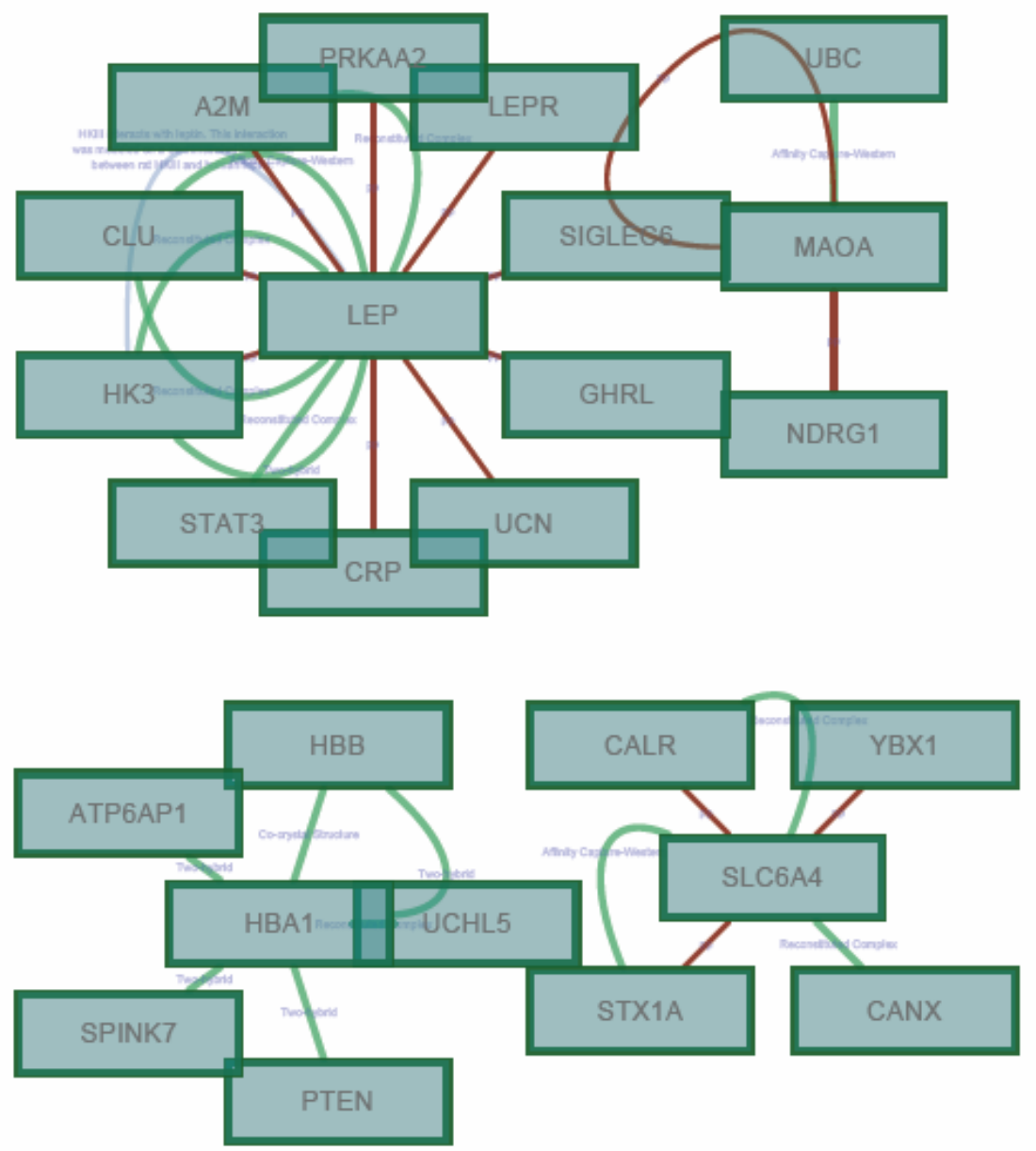

Fig. 6. Illustration and visualization of the JuB-insomnia interactome network. This sub network represents a co regulated unit containing 25 nodes and 31 edges. The interaction map includes four key genes and their neighbouring genes

between the diseases and genes were from the OMIM (http://www.ncbi.nlm.nih.gov/omim). Cytoscape has being developed for reconstruction and visualization of networks of insomnia. Nodes (represented as circles) in the interactome correspond to genes, and edges (connecting lines) represent documented interactions. Cytoscape is a desktop Java application and source code for Cytoscape 2.8 are available for download from http://cytoscape.org. Its default annotations are parsed from the GO information available from NCBI (http://www.ncbi.nlm.nih.gov/Ftp/). Probably the most stable one is the Entrez GeneID, which is the unique identifier for a gene in NCBI's Entrez Gene database.

\section{Results}

\section{Visualization in Cytoscape}

For pathway visualization and analysis of networks, we used open-source Cytoscape version 2.8 software (see Fig. 1). In Cytoscape, networks are represented as graphs where the nodes are the entities (e.g. genes, proteins) and the edges their interactions (e.g. reactions). For the visualization of mutagenesis in the context of biological networks, we developed three different node attribute files and two visual style files that can easily be imported into Cytoscape. This benchmark was run on a standard desktop computer (4GHz Pentium intel with $2 \mathrm{~GB}$ of memory running Windows XP).

\section{Insomnia multilayer network reconstruction}

Taking the closely-connected and co-expressed differentially expressed genes, for the insomnia network shown in Fig. 3-5, with 77 nodes and 96 edges. Seven of them are significant frequencies in insomnia and are likely to be responsible for driving the initiation, progression, or maintenance of disease. We find that 7 genes considered linked because they had close relationship and suggested as novel insomnia susceptibility genes using the Cytoscape visualization software. The 7 perturbed genes in the network has strong connections to the genes. This is indicated by the thick edges between these nodes. 


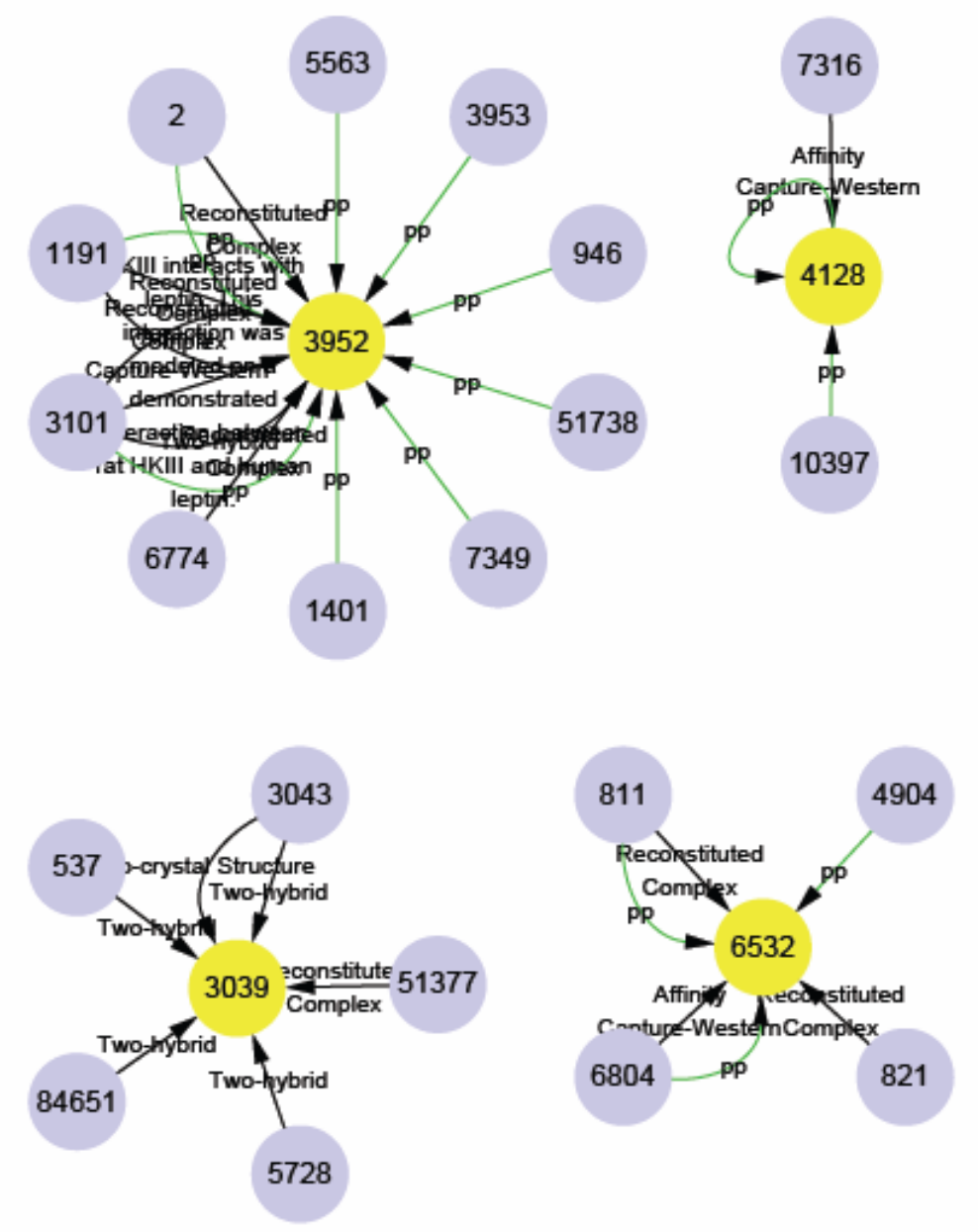

Fig. 7. A schematic displaying genes associated with the JuB-insomnia interactome. Central nodes (Yellow) of the interaction network were used to illustrate the gene expression obtained and represents significant change in expression. The connections between molecules show molecular interactions identified in the JuB -insomnia interactome. Gene expression illustrated in coloured nodes was selected with a $p<0.05$ value

\section{JuB-insomnia network model}

A useful tool to search a network between drugs or diseases is also available and the network type could be NCBI's Entrez Gene, KEGG, HPRD target network. Fig. 6 and 7 denotes the relationship between $\mathrm{a} J u B$ and insomnia disease, for a JuB network of 25 nodes and 31 edges. Main pathway information, 4 target-genes is observed in the Cytoscape-based network with significant P-values. These closely connected and co-expressed differentially expressed genes in genome wide networks are regarded as the signatures of the JuB underlying targets. Regulatory sub-network of target-genes on JuB through network pharmacology was constructed, and shown in Fig. 8.

\section{Discussion}

Deciphering the genetic basis of human complex diseases is an important goal of biomedical research. With the rapid progress of the cross-disciplines, the shift from the conceptual disease to the systematic drug-disease network is required for deeply understanding the molecular basis of mutual interrelationships among drug and disease systems. Recently, computational SysBiomics approaches have been enhancing our understanding of various aspects of complex diseases, including the identification of disease-related genes or the system mechanisms in diseases (Zhang, Sun, Yang, \& Wang, 2012). Related methods have been successfully applied for analyzing many physiological processes, such as type 2 diabetes, immunology, breast cancer metastasis and drug response (Burga, Casanueva, \& Lehner, 2011; Silva-Rocha \& de Lorenzo, 2011; Ma, Tan, Zhang, Wang, Ding, \& Li, 2010; Telesco, Shih, Jia, \& Radhakrishnan, 2011). Interaction network models are crucially important for disease processes. Many of the important properties of 

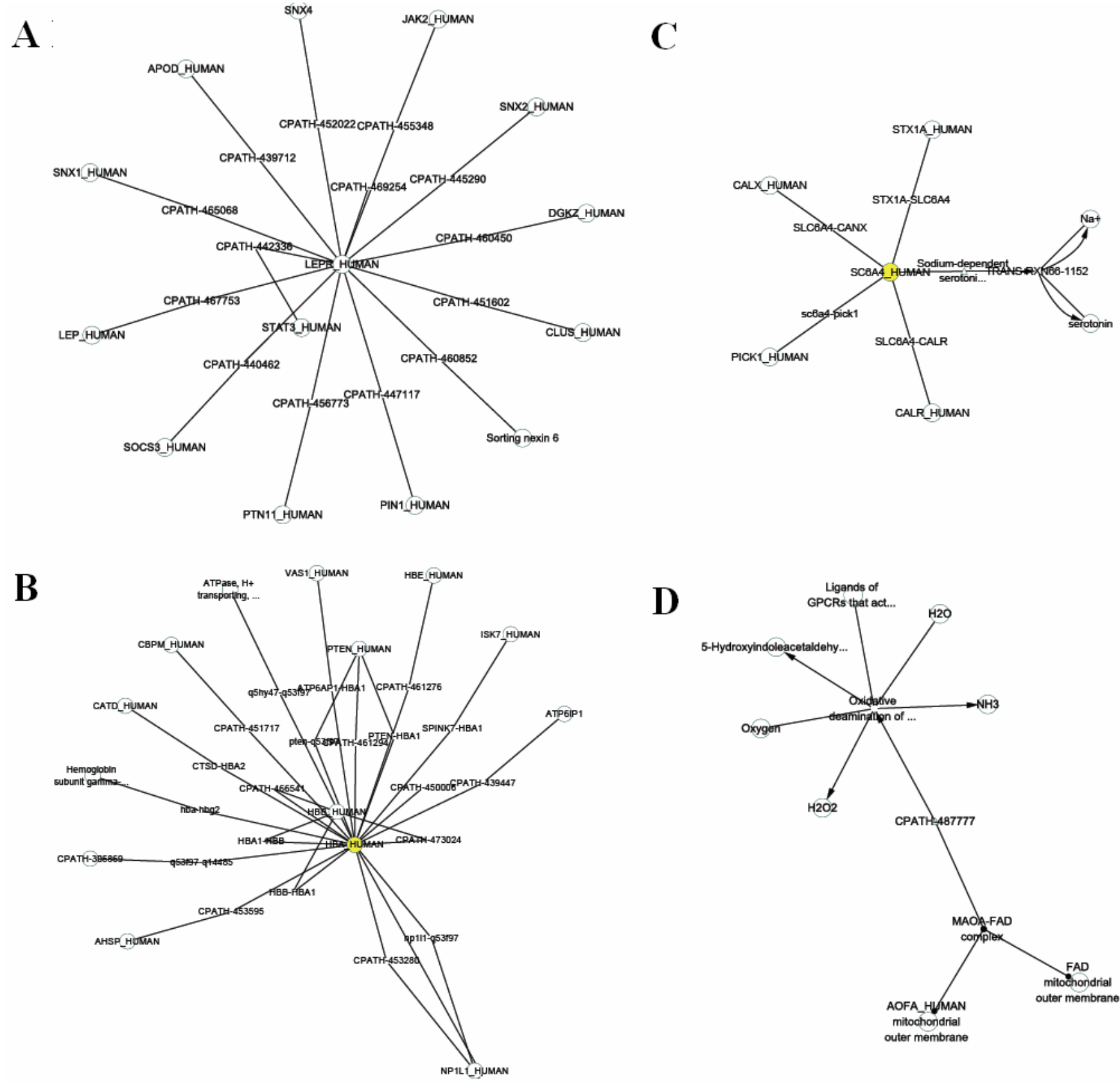

Fig. 8. Regulatory sub-network of target-genes on JuB through network pharmacology. A: LEP; B: HBA1; C: SLC6A4; D: MAOA

biological systems emerge as a result of the interactions among genes and among their protein products. Genes and the proteins they encode participate in gene-gene, gene-protein, and protein-protein interactions to mediate a wide variety of biological processes. Cytoscape is a bioinformatics software platform for visualizing molecular interaction networks and integrating these interactions with gene expression profiles and other state data. Cytoscape that can generate a putative protein-protein interaction network for target genomes, make the creation of protein-protein interaction network predicting tools possible. Its central organizing principle is a network graph, with biological entities (e.g. genes, proteins) represented as nodes and biological interactions represented as edges between nodes. Data are integrated with the network using attributes, which map nodes or edges to specific data values such as gene expression levels or protein functions. These features provide a mechanism for expressing relationships between sets of data while simultaneously visualizing the integrated results. In our study, to illustrate the utility of Cytoscape to researchers working with insomnia models, we have used the software 
to explore insomnia expression data in the context of biological network information. of note, Cytoscape successfully provided us with valuable clues for identification of drug-target interactions on a large scale and predicted and identified drug-target interactions.

Insomnia is the most common sleep disturbance with difficulty initiating or maintaining sleep, along with impaired daytime function (Shimomura, 2010). There is a high prevalence of insomnia in a variety of negative consequences. A meta-analysis of studies on sedative-hypnotics drugs caused a number of side effects, including habituation, physical and psychological dependency, daytime psychomotor and cognitive impairment, daytime drowsiness or anxiety, and rebound insomnia upon the abrupt discontinuation of sedative hypnotic therapy (Beland et al., 2010). In contrast, JuB, a classic natural product, has been efficiently used for insomnia relief in Asia, although its mechanism remains unclear. The existence of interactions between drugs and metabolites suggests a potential way to discover drug targets. A promising approach in drug target discovery involves the integration of available metabolites data through mathematical modelling and data mining. Significant work has been done on drug discovery, however, few papers were discussed with the metabolic network. This study was designed to further elucidate the underlying mechanism of JuB on sleep regulation from the metabolic pathways in a global view.

Biological networks analysis of the target metabolites from the insomnia is becoming increasingly important for assessing inherent network properties in reconstructed biochemical reaction networks. At present study, we have utilized biological networks approach further to delineate insomnia and JuB treatment. Of note, 4 differentially expressed genes were observed. LEP (GeneID 3952) encodes a protein that is secreted by white adipocytes, and which plays a major role in the regulation of body weight (Fig. 8 A). This protein, which acts through the leptin receptor, functions as part of a signalling pathway that can inhibit food intake and/or regulate energy expenditure to maintain constancy of the adipose mass. This protein also has several endocrine functions, and is involved in the regulation of immune and inflammatory responses, hematopoiesis, angiogenesis and wound healing. HBA1 (GeneID 3039) encode ghrelin-obestatin preproprotein, which generates ghrelin and obestatin (Fig. 8 B). Ghrelin is an endogenous ligand for the growth hormone secretagogue receptor and is involved in regulating growth hormone release. Obestatin was an endogenous ligand for the G protein-coupled receptor GPR39. Recent reports show that obestatin is involved in inhibiting thirst and anxiety, improving memory, regulating sleep, affecting cell proliferation, and increasing the secretion of pancreatic juice enzymes. SLC6A4 (GeneID 6532) encodes an integral membrane protein that transports the neurotransmitter serotonin from synaptic spaces into presynaptic neurons (Fig. 8C). The encoded protein terminates the action of serotonin and recycles it in a sodium-dependent manner. This protein is a target of psychomotor stimulants, such as amphetamines and cocaine, and is a member of the sodium:neurotransmitter symporter family. A repeat length polymorphism in the promoter of this gene has been shown to affect depression-susceptibility in people experiencing emotional trauma. MAOA (GeneID 4128) encodes monoamine oxidase $\mathrm{A}$, an enzyme that degrades amine neurotransmitters, such as dopamine, norepinephrine, and serotonin (Fig. 8, D). The protein localizes to the mitochondrial outer membrane.

The dominant paradigm in drug discovery is the concept of designing maximally selective drug targets. However, many effective drugs act via modulation of multitargets rather than single targets. Advances in systems biology are revealing that integrated network biology and polypharmacology holds the promise of expanding the current opportunity space for drug targets (Hopkins, 2008). Identification of drug targets is one of the major tasks in drug discovery (Raman, Vashisht, \& Chandra, 2009; Gu, Chen, Li, \& Li, 2010). More importantly, our methods extend the candidate target proteins to a genome-wide scale, which greatly enlarges the number of known targets. Our method benefits from current knowledge such as the known drug-target interactions, which provide us with golden standards for understanding drug mechanisms. Topological properties in networks reflect certain basic characteristics of biological systems. Together with known drug-target interactions, such information makes it possible to relate pharmacological space with genomic space. Thus, we believe that combining the integration of multi-dimensional information in pharmacological space and genomic space gains advantages in target identification information which could help to generate further drug discovery. Drug target is a key molecule involved in a particular metabolic or signalling pathway that is specific to a disease condition. Drugs can be designed to modify the functioning of the pathway in the diseased state by inhibiting a key molecule, or to enhance the normal pathway by promoting specific molecules that may have been affected in the diseased state and can influence the whole metabolic system by targets. Identification and validation of drug target is the essential first step in new drug discovery and development. Discovery of drug targets through network pharmacology analysis promises to be a useful and novel approach in this direction. Therefore, there is a strong incentive to develop new methods capable of discovering these potential target metabolites efficiently. Of note, we have characterized 4 specific genes relevant for drug target discovery and found drug-target interaction networks involve receptors, neurotransmitter, enzymes, 
signal transduction. Further experiments can be done to validate these targets. It provided strong evidence that the hypnotic effect of JuB occurred at the level of global network.

In the results, we demonstrated the usefulness of our proposed method for the prediction of the target interaction networks. We have constructed the first interaction network of the insomnia by using pattern recognition approach. These potential targets are promising for further experimental validation. Our study highlights the importance of network pharmacology as a potential tool for uncovering pathways to predict and discover drug targets, provide insights into drug action mechanisms and enable us to increase research productivity throughout the drug discovery and development process.

\section{Conclusions}

System networks that are a central paradigm in biology will help us in identifying new drug targets which in turn will generate more in-depth understanding of the mechanism of diseases and thus provide better guidance for drug discovery. Thus network-based pharmacology is emerging as an important paradigm for analysis of biological systems. A major strength of visualizing gene expression data layered onto interaction networks is that the networks contain large numbers of molecular relationships. Novel relationships between groups of co-regulated molecules can be rapidly identified, frequently linked through molecules that are not differentially expressed but nevertheless are important components of the overall functional network. In this paper, we present an integrated approach to predict targets for approved anti-insomnia JuB by exploring network pharmacology. Insomnia networks from our analysis were constructed with the aid of Cytoscape software. It also implies the significant contribution of network to predict drug targets. Finally, we apply our method to predict new and possible targets for insomnia and JuB drug. Analyzing the topology of the network, we have detected 4 potential drug targets and predicted the major interactome network of insomnia by using validated Cyoscape method. The identified targets were found to encompass a variety of pathways related to the HBA1, LEP, SLC6A4 and MAOA. Perturbed genes tend to be highly coexpressed and functionally coherent and we have used this property for predicting drug targets and associating novel functions to drug. Through networks analysis, the predicted targets may be involved in insomnia-associated biological networks. Indeed, future studies in human populations with insomnia will be needed to validate the biomarkers. Our findings also show that JuB exhibited preventive efficacy against insomnia by adjusting multiple pathways to their normal state, may be mediated through receptor, neurotransmitter, enzymes, and signal transduction. Thus, in practice, the integration involves the mapping to the nodes of insomnia network and the mapping of their functional effect to the edges of a network, for their use in the visualization and dynamic analysis. We expect that this approach will help in the study of the functional impact of insomnia-associated pathways, which ultimately will lead to a better understanding of the mechanisms underlying complex diseases.

\section{Acknowledgments}

This work was supported by grants from the Key Project of Special Foundation of Technology Innovation of Harbin (Grant No.2012RFQYS038), Key Program of Natural Science Foundation of State (Grant No. 81302905), Foundation of Heilongjiang University of Chinese Medicine (Grant no. 201209).

\section{References}

Beland, S. G., Preville, M., Dubois, M. F., Lorrain, D., Grenier, S., Voyer, P., Perodeau, G., \& Moride, Y. (2010). Benzodiazepine use and quality of sleep in the community-dwelling elderly population. Aging \& Mental Health, 14, 843-850. [PubMed: 20658372] doi:10.1080/13607861003781833

Burga, A., Casanueva, M. O., \& Lehner, B. (2011). Predicting mutation outcome from early stochastic variation in genetic interaction partners. Nature, 480, 250-253. [PubMed: 22158248] doi:10.1038/nature10665

Cline, M. S., Smoot, M., Cerami, E., Kuchinsky, A., Landys, N., Workman, C., ... Bader, G. D. (2007). Integration of biological networks and gene expression data using Cytoscape. Nature Protocols, 2, 2366-2382. [PubMed: 17947979] doi:10.1038/nprot.2007.324

Fang, X. Sh., Hao, J. F., Zhou, H. Y., Zhu, L. X., Wang, J. H., \& Song, F. Q. (2010). Pharmacological studies on the sedative-hypnotic effect of Semen Ziziphi spinosae (Suanzaoren) and Radix et Rhizoma Salviae miltiorrhizae (Danshen) extracts and the synergistic effect of their combinations. Phytomedicine. 17, 75-80. [PubMed: 19682877] doi:10.1016/j.phymed.2009.07.004

Gilchrist, M., Thorsson, V., Li, B., Rust, A. G., Korb, M., Roach, J. C., ... Aderem, A. (2006). Systems biology approaches identify ATF3 as a negative regulator of Toll-like receptor 4. Nature, 441, 173-178. [PubMed: 16688168] doi:10.1038/nature04768

$\mathrm{Gu}$ J., Chen, Y., Li, S., \& Li, Y. (2010). Identification of responsive gene modules by network-based gene clustering and extending: application to inflammation and angiogenesis. BMC Systems Biology, 4, 47. [PubMed: 20406493] doi:10.1186/1752-0509-4-47

Hopkins, A. L. (2008). Network pharmacology: the next paradigm in drug discovery. Nature Chemical Biology, 4, 682-690. [PubMed: 18936753] doi:10.1038/nchembio.118

Janga, S. C., \& Tzakos, A. (2009). Structure and organization of drug-target networks: insights from genomic approaches for drug discovery. Molecular Biosystematics, 5, 1536-1548. [PubMed: 19763339]

Ma, T., Tan, C., Zhang, H., Wang, M., Ding, W., \& Li, S. (2010). Bridging the gap between traditional Chinese medicine and systems biology: the connection of Cold Syndrome and NEI network. Molecular Biosystematics, 6, 613-619. [PubMed: 20237638] doi:10.1039/b914024g 
Mahowald, M. W. \& Schenck, C. H. (2005). Insights from studying human sleep disorders. Nature, 437, 1279-1285 doi:10.1038/nature04287 [PubMed: 16251953]

Mestres, J., Gregori-Puigjané, E., Valverde, S., \& Solé, R. V. (2009). The topology of drug-target interaction networks: implicit dependence on drug properties and target families. Molecular Biosystematics, 5, 1051-1057. [PubMed: 19668871]

Raman, K., Vashisht, R., \& Chandra, N. (2009). Strategies for efficient disruption of metabolism in Mycobacterium tuberculosis from network analysis. Molecular Biosystematics, 5, 1740-1751. [PubMed: 19593474] doi:10.1039/b905817f

Richey, S. M., \& Krystal, A. D. (2011). Pharmacological advances in the treatment of insomnia. Current Pharmaceutical Design, 17, 1471-1475. [PubMed: doi:10.2174/138161211796197052

Sarris, J., \& Byrne, G. J. (2011). A systematic review of insomnia and complementary medicine. Sleep Medicine Reviews, 15, 99-106. [PubMed: 20965131] doi:10.1016/j.smrv.2010.04.001

Shannon, P., Markiel, A., Ozier, O., Baliga, N. S., Wang, J. T., Ramage, D., ... Ideker, T. (2003). Cytoscape: a software environment for integrated models of biomolecular interaction networks. Genome Research, 13(11), 2498-504 doi:10.1101/gr.1239303 [PubMed: 14597658]

Shimomura, K., Lowrey, P. L., Vitaterna, M. H., Buhr, E. D., Kumar, V., Hanna, P., ... Takahashi, J. S. (2010). Genetic suppression of the circadian Clock mutation by the melatonin biosynthesis pathway. Proceedings of the National Academy of Sciences of the United States of America, 107, 8399-8403. [PubMed: 20404168] doi:10.1073/pnas.1004368107

Silva-Rocha, R., \& de Lorenzo, V. (2011). Implementing an OR-NOT (ORN) logic gate with components of the SOS regulatory network of Escherichia coli. Molecular Biosystematics, 7, 2389-2396. [PubMed: 21584342] doi:10.1039/c1mb05094j

Tamble, C. M., St Onge, R. P., Giaever, G., Nislow, C., Williams, A. G., Stuart, J, M., \& Lokey, R. S. (2011). The synthetic genetic interaction network reveals small molecules that target specific pathways in Sacchromyces cerevisiae. Molecular Biosystematics, 7, 2019-2030. [PubMed: 21487606] doi:10.1039/c0mb00298d

Telesco, S. E., Shih, A. J., Jia, F., \& Radhakrishnan, R. (2011). A multiscale modeling approach to investigate molecular mechanisms of pseudokinase activation and drug resistance in the HER3/ErbB3 receptor tyrosine kinase signaling network. Molecular Biosystematics, 7, 2066-2080. [PubMed: 21509365] doi:10.1039/c0mb00345j

Vecsey, C. G., Baillie, G. S., Jaganath, D., Havekes, R., Daniels, A., Wimmer, M., ... Abel, T. (2009). Sleep deprivation impairs cAMP signalling in the hippocampus. Nature, 461, 1122-1125. [PubMed: 19847264] doi:10.1038/nature08488

Wang, L., Zhou, G. B., Liu, P., Song, J. H., Liang, Y., Yan, X. J., ... Chen, Z. (2008). Dissection of mechanisms of Chinese medicinal formula Realgar-Indigo naturalis as an effective treatment for promyelocytic leukemia. Proceedings of the National Academy of Sciences of the United States of America. 105, 4826-4831. [PubMed: 18344322] doi:10.1073/pnas.0712365105

Wu, X., Jiang, R., Zhang, M. Q., \& Li, S. (2008). Network-based global inference of human disease genes. Molecular Systems Biology. 4, 189. [PubMed: 18463613] doi:10.1038/msb.2008.27

Yang, B., Zhang, A., Sun, H., Dong, W., Yan, G., Li, T., \& Wang, X. (2012). Metabolomic study of insomnia and intervention effects of Suanzaoren decoction using ultra-performance liquid-chromatography/electrospray-ionization synapt high-definition mass spectrometry. Journal of Pharmaceutical and Biomedical Analysis, 58, 113-124. [PubMed: 22019702] doi:10.1016/j.jpba.2011.09.033

Yao, X., Hao, H., Li, Y., \& Li, S. (2011). Modularity-based credible prediction of disease genes and detection of disease subtypes on the phenotype-gene heterogeneous network. BMC Systems Biology, 5, 79. [PubMed: 21599985] doi:10.1186/1752-0509-5-79

Zhang, A., Sun, H., Yang, B., \& Wang, X. (2012). Predicting new molecular targets for rhein using network pharmacology. BMC Systems Biology, 6, 20. PMID: 22433437 doi:10.1186/1752-0509-6-20

Zhang, B., Shi, Z., Duncan, D. T., Prodduturi, N., Marnett, L. J., \& Liebler, D. C. (2011). Relating protein adduction to gene expression changes: a systems approach. Molecular Biosystematics, 7, 2118-2127. [PubMed: 21594272] doi:10.1039/c1mb05014a

Zhao, J., Li, S. P., Yang, F. Q., Li, P., \& Wang, Y. T. (2006). Simultaneous determination of saponins and fatty acids in Ziziphus jujuba (Suanzaoren) by high performance liquid chromatography-evaporative light scattering detection and pressurized liquid extraction. Journal of Chromatography $A$, 1108, 188-194. doi:10.1016/j.chroma.2005.12.104

[PubMed:16458908]

Zou, J., Luo, S. D., Wei, Y. Q., \& Yang, S. Y. (2011). Integrated computational model of cell cycle and checkpoint reveals different essential roles of Aurora-A and Plk1 in mitotic entry. Molecular Biosystematics, 7, 169-179. [PubMed: 20978655] doi:10.1039/c0mb00004c 